\title{
VARIAÇÃO E ASSOCIAÇÃO DE CARACTERÍSTICAS LIGADAS A FORMAÇÃO DO GRÃO DE GENÓTIPOS DE TRIGO
}

\author{
Jorge Luiz Nedel ${ }^{1,3 *}$; Cirilo Nelson Enrique Molas González²; Silmar Teichert Peske ${ }^{1,3}$ \\ ${ }^{1}$ Depto. de Fitotecnia - FAEM/UFPel, C.P. 354 - CEP: 96001-970 - Pelotas, RS. \\ ${ }_{3}^{2}$ DISE - Ministério da Agricultura - Assunção, Paraguai. \\ ${ }^{3}$ Bolsista do CNPq. \\ *e-mail: jlnedel@ufpel.tche.br
}

RESUMO: O rendimento de trigo (Triticum aestivum L.) é estabelecido pelo número de grãos produzidos por unidade de área e pelo peso do grão. Este é determinado pela taxa e duração do desenvolvimento do grão. A taxa e a duração do enchimento do grão foram determinados em sete genótipos de trigos brasileiros, com o objetivo de avaliar a variação genotípica para estes caracteres e a relação dos mesmos com parâmetros agronômicos. Variações genotípicas significativas foram observadas, tanto para a taxa como para a duração de enchimento da grão. A taxa e a duração de enchimento do grão variaram de 0,077 a $0,089 \mathrm{mg} / \mathrm{sem} / \mathrm{gráus}$ dias acumulados e de 27 a 31 dias, respectivamente. A taxa e não a duração de enchimento estava associada ao peso do grão. A seleção de genótipos com maior peso de grão pode levar à obtenção de cultivares de trigo precoces, com maior taxa de enchimento de grãos e adequadas a um sistema de duas culturas anuais.

Palavras chave: Triticum aestivum, taxa de enchimento do grão, peso do grão

\section{VARIATION AND ASSOCIATION OF KERNEL GROWTH CHARACTERISTICS OF WHEAT GENOTYPES}

\begin{abstract}
Yield of wheat seed is a function of the number of grains produced per unit area and grain weight. Grain weight is affected by the rate and duration of the kernel growth. The objective of this experiment was to measure the rate and duration of grain filling of seven brazilian wheat genotypes in order to evaluate genotypic variation for these characters and to examine the relationship between rate and duration of grain filling, and other agronomic parameters. Significant genotypic variation for rate and duration of seed filling was observed. Rate of seed filling varied from 0.077 to $0.089 \mathrm{mg} / \mathrm{seed} / \mathrm{accumulated}$ growing degree days, while duration of grain filling varied between 27 to 31 days. Rate, but not duration of grain filling, was correlated with grain weight. Kernel weight may be an appropriate selection criteria to be used by breeders to select early maturing wheat genotypes for higer grain filling rate.
\end{abstract}

Key words: Triticum aestivum, rate of grain filling, kernel weight

\section{INTRODUÇÃO}

Na Região Sul de cultivo de trigo (sul do Paraná, Santa Catarina e Rio Grande do Sul) o trigo está mais sujeito a estresses durante o período de enchimento do grão, o qual é causado, principalmente, por excesso de umidade, baixa insolação, altas temperaturas e doenças da parte aérea. Esses tipos de estresses aceleram a redução da área foliar verde da planta, diminuindo o período de enchimento do grão. Após o número de grãos ser estabelecido, o rendimento do trigo passa a ser proporcional ao peso do grão (Wiegand \& Cuellar, 1981), o qual é função da taxa e do tempo de enchimento do grão (Darroch \& Baker, 1990).

A taxa de enchimento do grão é dependente do número de células formadas na grão em desenvolvimento durante as primeiras duas semanas após a antese (Brocklehurst, 1977). Essa taxa é aumentada moderadamente com a elevação da temperatura, enquanto a duração do enchimento é significativamente reduzida (Wych et al., 1982; Van Sanford, 1985). O aumento na taxa, no entanto, não é suficiente para compensar a perda no peso do grão em conseqüência da redução no número de dias de enchimento (Caley et al., 1990). A baixa insolação, durante o período de enchimento do grão, causa um decréscimo na taxa de enchimento do grão, em conseqüência da redução na fotossíntese atual. Em tais situações, aquela passa a depender mais da capacidade da cultivar de remobilizar carboidratos de outras partes da planta, especialmente do colmo (Palta et al., 1994). 
A precocidade é uma característica fundamental para que uma cultivar de trigo possa se incorporar, de forma expressiva, ao sistema de cultivo utilizado na Região Sul, onde, geralmente, pelo menos duas safras são obtidas por ano, por sucessão de cultivo. Conseqüentemente, para que o rendimento do trigo possa ser aumentado e a precocidade mantida, os programas de melhoramento deste cereal devem considerar a seleção de genótipos com maior taxa de enchimento de grão. A variabilidade genética para esta característica e sua associação positiva com o peso do grão, tem sido observada entre cultivares de trigo de primavera (Bruckner \& Frohberg, 1987; Darroch \& Baker, 1990; Hunt et al., 1991; Darroch \& Baker, 1995). Sofield et al. (1977) e Gabeyehou et al. (1982) verificaram, também, correlação entre duração do enchimento e peso do grão. Portanto, aumentos no peso das grãos poderiam resultar de maiores períodos de enchimento. Já, Gabeyehou et al. (1982) observaram baixa associação entre taxa e duração de enchimento do grão, e sugeriu que seria possível a seleção para aumento na taxa de enchimento e peso do grão sem aumento na duração do período de enchimento.

Os objetivos deste estudo foram de: 1- avaliar a variabilidade entre genótipos de trigos brasileiros, com características diferentes, para taxa e duração de enchimento de grãos e 2examinar a associação entre parâmetros relacionados com enchimento do grão e características agronômicas.

\section{MATERIAL E MÉTODOS}

Sete genótipos de trigo, escolhidos por apresentarem características de peso de grão diferentes, foram semeados no campo experimental da Fazenda da Palma, da Faculdade de Agronomia "Eliseu Maciel" de Pelotas - RS em 1994, para avaliação da taxa e duração de enchimento de suas grãos bem como suas características agronômicas.

$\mathrm{Na}$ condução do experimento a campo utilizou-se uma densidade de semeadura de 160 grãos viáveis por $\mathrm{m}^{2}$; uma adubação de base, 300 $\mathrm{kg} / \mathrm{ha}$ da fórmula 6-28-20 e de cobertura, por ocasião do perfilhamento, de $50 \mathrm{~kg} / \mathrm{ha}$ de nitrogênio na forma de uréia. As ervas daninhas foram controladas, de forma manual, no início do desenvolvimento da cultura. Cuidados especiais se teve no controle das doenças da parte aérea. Realizaram-se três aplicações do fungicida Tebuconazole: a primeira durante o alongamento do trigo, correspondendo ao estádio 7 da escala de Feeks e Large (Large, 1954), a segunda e terceira no inicio e fim da floração, correspondendo ao estádio 10.5.1 e 10.5.3, respectivamente.

As dimensões das parcelas foram de $5,0 \times 1,5 \mathrm{~m}$, com espaçamento de $0,25 \mathrm{~m}$ entre as linhas. Por ocasião da antese, marcaram-se em cada parcela, 80 espigas que apresentavam, na região central, extrusão recente das anteras. Todas as espigas marcadas eram de perfilhos principais. Amostras de cinco espigas marcadas foram colhidas a intervalos menores (dois dias) nas primeiras colheitas, iniciando no quinto dia após antese, e maiores (cinco dias) nas últimas. As espigas foram secas em estufa com circulação de ar, a temperatura de $60^{\circ} \mathrm{C}$, por 48 hs. Dos grãos do terço médio das espigas de cada amostra, determinou-se o número e o peso seco, para o cálculo do peso médio do grão.

Os graus dias acumulados (GDA) foram utilizados como escala de tempo durante o enchimento do grão, porque a taxa de desenvolvimento da planta de trigo é determinada, predominantemente, pela temperatura (Bauer et al., 1984). No cálculo dos graus dias utilizou-se $5^{\circ} \mathrm{C}$ como temperatura base. A taxa de enchimento do grão, expressa em miligramas de matéria seca por grão por graus dias acumulados, foi calculada dividindose o peso seco do grão na maturidade fisiológica (MF) pelos graus dias acumulados durante 0 período compreendido entre a antese até a MF. Este período (MF) foi definido como o necessário para a grão atingir o máximo de matéria seca.

Para determinar o índice de colheita (relação entre peso do grão e peso da palha), foram colhidas sete plantas, da área destinada para determinação do rendimento.

O rendimento de grãos foi determinado colhendo-se uma área de $3 \mathrm{~m}^{2}$ por parcela. 0 número de espigas por $\mathrm{m}^{2}$ foi estimado contando -se o número de espigas que apresentavam grãos em dois metros de linha na área destinada à avaliação do rendimento. $\mathrm{O}$ número de grãos por espiga e o peso do grão (como componente do rendimento) foram calculados utilizando-se as plantas colhidas para a determinação do índice de colheita.

O delineamento experimental adotado foi de blocos casualizados, com oito repetições. As médias foram comparadas pelo teste de Duncan a 0,05 de probabilidade. Para cada parcela, a relação entre peso do grão e o número de dias após antese foi descrita por uma equação polinomial quadrática. 


\section{RESULTADOS E DISCUSSÃO}

Os resultados referentes aos parâmetros estudados encontram-se na TABELA 1. O teste de $\mathrm{F}$ mostrou diferenças significativas até $5 \%$ de probabilidade para todas as variáveis, exceto o rendimento de grãos.

Verificou-se que a duração média do período da antese até a maturidade fisiológica foi de 28 dias e da emergência até esta, 113 dias, correspondendo a 393 e 1230 graus dias acumulados, respectivamente. A duração média de ambos os períodos, foi inferior ao normalmente verificado, em torno de 39 dias e 127 dias, respectivamente, para estas cultivares nas condições de cultivo no Rio Grande do Sul. Essa redução deveu-se, principalmente, à menor precipitação pluviométrica ( 74 e $77 \%$ da normal) ocorrida durante os dois estádios de desenvolvimento do trigo no ano de 1994.

A taxa média de enchimento do grão foi de $0,084 \mathrm{mg} / \mathrm{graus}$ dias acumulados (TABELA 1). Esta taxa de enchimento de grão é semelhante às observadas por Gebeyehou et al. (1982), porém bem superior à reportada por Bruckner \& Frohberg (1987). O peso médio do grão na maturidade fisiológica foi de $35,3 \mathrm{mg}$, indicando que, apesar da redução no número de dias de enchimento das mesmas e, em conseqüência da contribuição da fotossíntese atual, a remobilização de carboidratos das demais estruturas da planta foi suficiente para garantir a produção de uma grão com peso normal.

Entre os genótipos avaliados, a linhagem PF 889199 foi a que apresentou a maior taxa de acúmulo de matéria seca na grão $(0,089 \mathrm{mg} / \mathrm{graus}$ dias $)$ enquanto a cultivar EMBRAPA 16 a menor $(0.077 \mathrm{mg} / \mathrm{graus}$ dias $)$. A linhagem PF 889199 também foi a que apresentou o maior peso médio das grãos $(37,7 \mathrm{mg})$ enquanto a cultivar EMBRAPA 16, o menor $(32,6 \mathrm{mg})$. Estes dados indicam que há uma substancial variação genotípica para taxa de enchimento e peso do grão. Esta resposta é indicativa de uma provável associação entre os dois parâmetros, confirmada pelo alto valor do coeficiente de correlação $(r=0,888 ; n=7)$ observado (TABELA 2). Estes resultados são consistentes com os obtidos por Van Sanford, (1985); Bruckner \& Frohberg, (1987). Aude et al. (1994) não observaram variabilidade para taxa de enchimento de grãos entre as cultivares de trigo brasileiras estudas.

A taxa de enchimento $\left(r=0,888^{* *} ; n=7\right)$ e não a duração em graus dias $(r=0,381 ; n=7)$ do enchimento de grão, esteve associada ao peso do grão (TABELA 2). Estes resultados sugerem que é possível aos melhoristas de trigo selecionarem materiais com uma maior taxa de enchimento sem aumentar o período de enchimento do grão. A alta correlação entre a taxa de enchimento e peso do grão indica,

TABELA 1 - Médias de parâmetros de enchimento do grão, componentes de rendimento e de características agronômicas, de sete genótipos de trigo avaliados em 1994. UFPel.

\begin{tabular}{|c|c|c|c|c|c|c|c|c|c|c|c|c|}
\hline \multirow{4}{*}{ Genótipos } & \multicolumn{3}{|c|}{$\begin{array}{l}\text { Parâmetros do enchimento } \\
\text { do grão }\end{array}$} & \multicolumn{3}{|c|}{$\begin{array}{l}\text { Componentes do } \\
\text { rendimentos }\end{array}$} & \multicolumn{6}{|c|}{ Características Agronômicas } \\
\hline & \multirow{3}{*}{$\frac{\text { Taxa }}{\text { mg/sem }}$} & Dura & ação & \multirow{3}{*}{$\begin{array}{l}\text { Peso do } \\
\text { grão } \\
\text { mg }\end{array}$} & \multirow{3}{*}{$\begin{array}{c}\text { Grãos/ } \\
\text { espiga } \\
\text { No. }\end{array}$} & \multirow{3}{*}{$\frac{\begin{array}{c}\text { Espigas/ } \\
\mathrm{m}^{2}\end{array}}{\text { No. }}$} & \multirow{3}{*}{$\begin{array}{l}\text { Rendi- } \\
\text { mento } \\
\mathrm{kg} / \mathrm{ha}\end{array}$} & \multirow{3}{*}{$\begin{array}{l}\text { Índice de } \\
\text { Colheita } \\
\text { índice }\end{array}$} & \multicolumn{4}{|c|}{ Ciclo } \\
\hline & & $\begin{array}{r}\text { Antese } \\
\mathrm{fi}\end{array}$ & latur. & & & & & & \multicolumn{2}{|c|}{$\begin{array}{c}\begin{array}{c}\text { Emergência à } \\
\text { Antese }\end{array} \\
\end{array}$} & \multicolumn{2}{|c|}{$\begin{array}{l}\text { Emergência à } \\
\text { Matur. fisiol. }\end{array}$} \\
\hline & & Dias & GDA & & & & & & Dias & GDA & Dias & GDA \\
\hline R 23 & 0 & 0 & b & 3 & 3 & b & 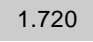 & $0,43 a$ & c & & $c$ & $1226,9 \mathrm{~b}$ \\
\hline 235 & $81 \mathrm{c}$ & ,13 b & 395,2 a & $36,2 a b$ & $34,5 a b$ & $225,5 \mathrm{a}$ & 1.747 & $0,38 \mathrm{c}$ & $84,13 \mathrm{bc}$ & 825,2 bc & $111,3 \mathrm{c}$ & $1220,0 \mathrm{~b}$ \\
\hline MBRP 16 & $077 d$ & $3,00 \mathrm{~b}$ & $392,4 \mathrm{~b}$ & $32,6 \mathrm{c}$ & $33,3 \mathrm{~b}$ & 243,5 a & 1.800 & 0,39 & 850 & $836,1 \mathrm{~b}$ & $113,0 \mathrm{~b}$ & $1228,5 b$ \\
\hline 24 & 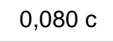 & $b$ & $a b$ & $33,0 \mathrm{~b}$ & $37,8 \mathrm{a}$ & $187,6 \mathrm{c}$ & 1.673 & 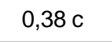 & $38 \mathrm{bc}$ & $28,4 \mathrm{bc}$ & 8 bc & 1221 \\
\hline PAR 41 & 086 a & $1,00 a b$ & $391,5 b$ & $37,5 \mathrm{a}$ & $34,8 a b$ & $217,5 a b$ & 1.953 & 0,35 e & $88,00 \mathrm{a}$ & 873,3 a & $119,0 a b$ & $1264,8 \mathrm{a}$ \\
\hline 869120 & $0,082 \mathrm{bc}$ & $27,88 \mathrm{~b}$ & 391,7 b & $34,4 \mathrm{~b}$ & $28,8 \mathrm{c}$ & $245,1 \mathrm{a}$ & 1.672 & $0,36 \mathrm{~d}$ & $84,88 \mathrm{bc}$ & 834,7 b & $112,6 \mathrm{bc}$ & 1226,4 \\
\hline 889199 & $0,089 \mathrm{a}$ & $27,00 \mathrm{c}$ & $396,0 \mathrm{a}$ & 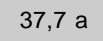 & $35,4 a b$ & $185,1 \mathrm{c}$ & 1.631 & $0,34 \mathrm{f}$ & 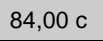 & 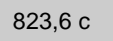 & $111,4 \mathrm{c}$ & $1219,6 \mathrm{~b}$ \\
\hline ura & 104 & 28,02 & 393,4 & 35,31 & 33,9 & 217,2 & 1.742 & 0,38 & 85,02 & 836,32 & 113,04 & 1229,77 \\
\hline V (\%) & 7,81 & 2,8 & 0,65 & 7,02 & 10,63 & 13,5 & 15.82 & 5,3 & 0,92 & 1,17 & 1,13 & 0,94 \\
\hline
\end{tabular}

${ }^{1} \mathrm{GDA}=$ Graus Dias Acumulados 
TABELA 2 - Coeficientes de correlação entre as médias de parâmetros de ciclo da planta, de enchimento do grão e de características agronômicas de sete genótipos de trigo $(n=7)$ avaliados em 1994. FAEM/UFPel.

\begin{tabular}{|c|c|c|c|c|c|c|c|c|c|c|c|c|}
\hline Parâmetros & $E-A$ & A-MF & E-MF & $\begin{array}{l}\text { E-A } \\
\text { GDA }\end{array}$ & $\begin{array}{l}\text { A-MF } \\
\text { GDA }\end{array}$ & $\begin{array}{l}\text { E-MF } \\
\text { GDA }\end{array}$ & $\begin{array}{c}\text { Taxa de } \\
\text { enchimento } \\
\text { GDA }\end{array}$ & $\begin{array}{l}\text { Peso do } \\
\text { grão }\end{array}$ & $\begin{array}{l}\text { Espigas/ } \\
\mathrm{m}^{2}\end{array}$ & $\begin{array}{l}\text { Grão/ } \\
\text { espiga }\end{array}$ & $\begin{array}{l}\text { Rendimento } \\
\text { de grãos }\end{array}$ & $\begin{array}{l}\text { Indice de } \\
\text { Colheita }\end{array}$ \\
\hline$E-A^{1}$ & - & 1,0 & $0,99^{* *}$ & 1,0 & $-0,685$ & $0,998^{* *}$ & 0,216 & 0,307 & 0,207 & $-0,035$ & $0,901^{* *}$ & $-0,248$ \\
\hline$A-M F^{2}$ & - & - & $0,999^{* *}$ & 1,0 & $-0,683$ & $0,998^{* *}$ & 0,216 & 0,307 & 0,207 & $-0,035$ & $0,901^{* *}$ & $-0,248$ \\
\hline $\mathrm{E}-\mathrm{MF}^{3}$ & - & - & - & 1,0 & $-0,704$ & $0,996^{\star *}$ & 0,199 & 0,286 & 0,221 & $-0,053$ & $0,896^{* *}$ & $-0,230$ \\
\hline E-A GDA & - & - & - & - & $-0,695$ & $0,997^{\star \star}$ & 0,208 & 0,295 & 0,210 & $-0,042$ & $0,896^{* *}$ & $-0,241$ \\
\hline A-MF GDA & - & - & - & - & - & $-0,637$ & 0,358 & 0,381 & $-0,610$ & 0,450 & $-0,576$ & $-0,029$ \\
\hline E-MF GDA & - & - & - & - & - & - & 0,265 & 0,364 & 0,171 & $-0,010$ & $0,899^{* *}$ & $-0,260$ \\
\hline \multicolumn{13}{|c|}{ Taxa de enchimento } \\
\hline GDA & - & - & - & - & - & - & - & $0,888^{\star \star}$ & $-0,537$ & 0,117 & $-0,045$ & $-0,540$ \\
\hline Peso do grão & - & - & - & - & - & - & - & - & $-0,325$ & 0,095 & 0,210 & $-0,413$ \\
\hline Espigas $/ \mathrm{m}^{2}$ & - & - & - & - & - & - & - & - & - & $-0,795^{\star}$ & 0,340 & 0,214 \\
\hline Grãos/espiga & - & - & - & - & - & - & - & - & - & - & 0,056 & $-0,714$ \\
\hline
\end{tabular}

Rendimento de

grãos

$\begin{array}{llll}- & - & - & -\end{array}$

${ }^{1} \mathrm{E}-\mathrm{A}=$ Emergência à Antese (dias)

${ }^{2} \mathrm{~A}-\mathrm{M}=$ Antese à Maturação Fisiológica (dias)

${ }^{3} \mathrm{E}-\mathrm{MF}=$ Emergência à Maturação Fisiológica (dias)

${ }^{4} \mathrm{E}-\mathrm{A}$ GDA = Emergência à Antese ( gráus dias acumulados)

também, que a seleção para maior peso de grão pode levar à seleção de materiais com maior taxa de enchimento. A redução do período reprodutivo (antese-maturação) sem reduzir o peso do grão é desejável, considerando que a planta e o grão ficariam por menos tempo expostas às condições adversas de clima, durante os estádios mais críticos da cultura, nas condições da Região Sul Brasileira de trigo. Com base nas correlações fenotípicas observadas, pode-se inferir que não há barreiras genéticas que poderiam impedir o desenvolvimento de genótipos de trigo com alto peso de grãos, com altas taxas e reduzidos períodos de enchimento das mesmas.

O peso do grão nos cereais, em geral, é um fator que tem grande influência sobre o vigor das grãos. Conseqüentemente, contribui para o desenvolvimento de plântulas mais vigorosas e um estabelecimento rápido no campo, facilitando, dessa forma, práticas culturais como controle de ervas daninhas (Nedel et al., 1996). Portanto, o desenvolvimento de genótipos que apresentam uma maior taxa de enchimento de grãos poderia, potencialmente, contribuir para que os mesmos apresentassem um vigor de desenvolvimento inicial maior. Característica esta muito importante, principalmente, para um sistema de cultivo onde se utiliza a semeadura direta.
Os dados mostram claramente que 0 genótipo IAPAR 41 requer um acúmulo maior de graus dias para iniciar o florescimento (TABELA 1) que os demais. Com relação ao período de enchimento do grão, embora a análise estatística indique diferenças significativas, do ponto de vista prático, pode-se considerar que os genótipos estudados apresentaram a mesma exigência, em termos de acúmulo de graus dias, para completar esta fase. Estes resultados reforçam a informação, já existente na literatura (Evans \& Wardlaw, 1976), de que o período da emergência à antese é o que, na grande maioria das situações, determina a diferença de ciclo entre genótipos de trigo. Os maiores coeficientes de associação verificados entre duração de dias e graus dias acumulados da emergência à antese, em relação ao ciclo do trigo (E-MF), confirmam que este é determinado pelo período que vai da emergência à antese.

Os genótipos não diferiram entre si no rendimento de grãos, os quais, por sua vez, foram relativamente baixos. A causa destes baixos rendimentos está, provavelmente, relacionada ao maior espaçamento entre linhas utilizado $(25 \mathrm{~cm})$ e ao baixo perfilhamento resultante da menor precipitação, o que culminou num menor número de espigas por área, mantendose, porém, dentro do esperado o peso de grão e o numero de grãos por espiga (TABELA 1). 
As altas associações observadas entre os parâmetros de ciclo e de rendimento (TABELA 2), provavelmente estejam relacionados à redução forçada daqueles, determinadas pelas condições de chuvas a que a cultura esteve exposta neste ano de cultivo.

O índice de colheita, apresentou diferenças entre os genótipos, sendo a cultivar BR 23 a mais alta $(0,43)$ e, o menor, o genótipo PF 889199 (0,34).

A equação de regressão polinomial quadrática (Figura 1) utilizada para descrever o desenvolvimento do grão, durante todo o período de enchimento, fornece uma boa descrição do enchimento do grão para os genótipos utilizados, tendo em vista que peso do grão e os dados de graus dias acumulados se ajustam ao modelo com um coeficiente de determinação $r^{2}=0,986$. Os resultados confirmam as observações de Wang et al. (1995) que, até aproximadamente 17 dias após a antese, ocorre fundamentalmente, divisão celular e, a partir daí, aumento na deposição de reservas no grão.

\section{CONCLUSÕES}

- Ocorre significativa variabilidade genética para taxa de enchimento de grãos entre genótipos de trigo avaliados.

- O peso de grão de trigo é um parâmetro adequado para obtenção de genótipos com maior taxa de enchimento de grão.

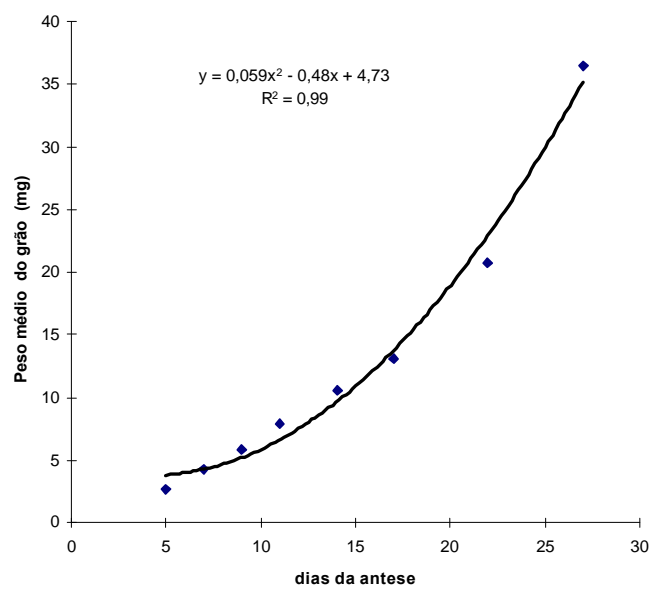

Figura1 - Peso médio e período de enchimento do grão da antese à maturação fisiológica de sete genótipos de trigo.

\section{REFERÊNCIAS BIBLIOGRÁFICAS}

AUDE, M.I.S.; MARCHESAN, E.; MAIRESSE, L.A.S.; BISOGNIN, D.A.; CIMA R.J.; ZANINI, W. Taxa de acúmulo de matéria seca e duração do período de enchimento de grão do trigo. Pesquisa Agropecuária Brasileira, v.29, n.10, p.1533-1539, 1994.

BAUER, A.; FRANK, A.B.; BLACK, A.L. Estimation of spring wheat leaf growth rates and anthesis from air temperature. Agronomy Journal, v.76, n.5, p.829-835, 1984.

BROCKLEHURST, P.A. Factors controlling grain wheight in wheat. Nature, v.266, p.348-349, 1977.

BRUCKNER, P.L.; FROHBERG, R.C. Rate and duration of grain fill in spring wheat. Crop Science, v.27, n.3, p.451-455, 1987.

CALEY, C.Y.; DUFFUS, C.M.; JEFFCOAT, B. Effects of elevated Temperature and Reduced Water Uptake on Enzymes of Starch Synthesis in Developing Wheat Grains. Australian Journal of Plant Physiology, v.17, p.431-439, 1990.

DARROCH, B.A.; BAKER, R.J. Grain filling in three spring wheat genotypes: Statistical analysis. Crop Science, v.30, n.3, p.525-529, 1990.

DARROCH, B.A.; BAKER, R.J. Two measures of grain filling in spring wheat. Crop Science, v.35, n.1, p.164-168, 1995.

EVANS, L.T.; WARDLAW, I.F. Comparative physiology of grain yields in cereals. Advances in Agronomy, v.28, p.301-359, 1976.

GEBEYEHOU, G.; KNOTT, D.R.; BAKER, R.J. Rate and duration of grain filling in durum wheat cultivars. Crop Science, v.22, n.2, p.337-340, 1982.

HUNT, L.A.; POORTEN, V.; PARARAJASINGHAM, $S$. Postanthesis temperature effects on duration and rate grain filling in some winter and spring wheats. Canadian Journal of Plant Science, v.71, p.609-617, 1991.

LARGE, E.C. Growth stages in cereals. Plant Pathology, v.3, p.128-129, 1954.

NEDEL, J.L.; ULLRICH, S.E.; PAN, W.L. Effect of seed size and protein content and $\mathrm{N}$ application timing on seedling vigor and gran yield of barley. Pesquisa Agropecuária Brasileira, v.31, n.2, p.113-119, 1996.

PALTA, J.A.; KOBATA, T.; TURNER, N.C.; FILLERY, I.R. Remobillzation of carbon and nitrogen in wheat as influenced by postanthesis water deficit. Crop Science, v.34, n.1, p.118-124, 1994.

SOFIELD, I.; EVANS, L.T.; COOK, M.G.; WARDLAW, I.F. Factors influencing rate and duration of grain filling in wheat. Australian Journal of Plant Physiology, v.4, p.785-797, 1977. 
VAN SANFORD, D.A. Variation in kernel growth characters among soft red winter wheats. Crop Science, v.25, n.4, p.626-630, 1985.

WANG, H.L.; PATRICK, J.W.; OFFLER, C.E. Assimilate delivery pathways in developing wheat grain and their implications for control of carbon transport. In: MADORE, M.A.; LUCAS, W.J. (Ed.) Carbon partitioning and source-sink interaction in plants. Rockville: American Society of Plant Physiologists, 1995. v.13, p.156-169.
WIENGAND, C.L.; CUELLAR, J.A. Duration of grain filling and kernel weight of wheat as affected by temperature. Crop Science, v.21, n.1, p.95-101, 1981.

WYCH, R.D.; McGRAW, R.L.; STUTHMAN, D.D. Genotype $x$ year interaction for lenght and rate of grain filling in oats. Crop Science, v.22, n.6, p.1025-1028, 1982.

Recebido para publicação em 18.11 .98 Aceito para publicação em 06.07.99 